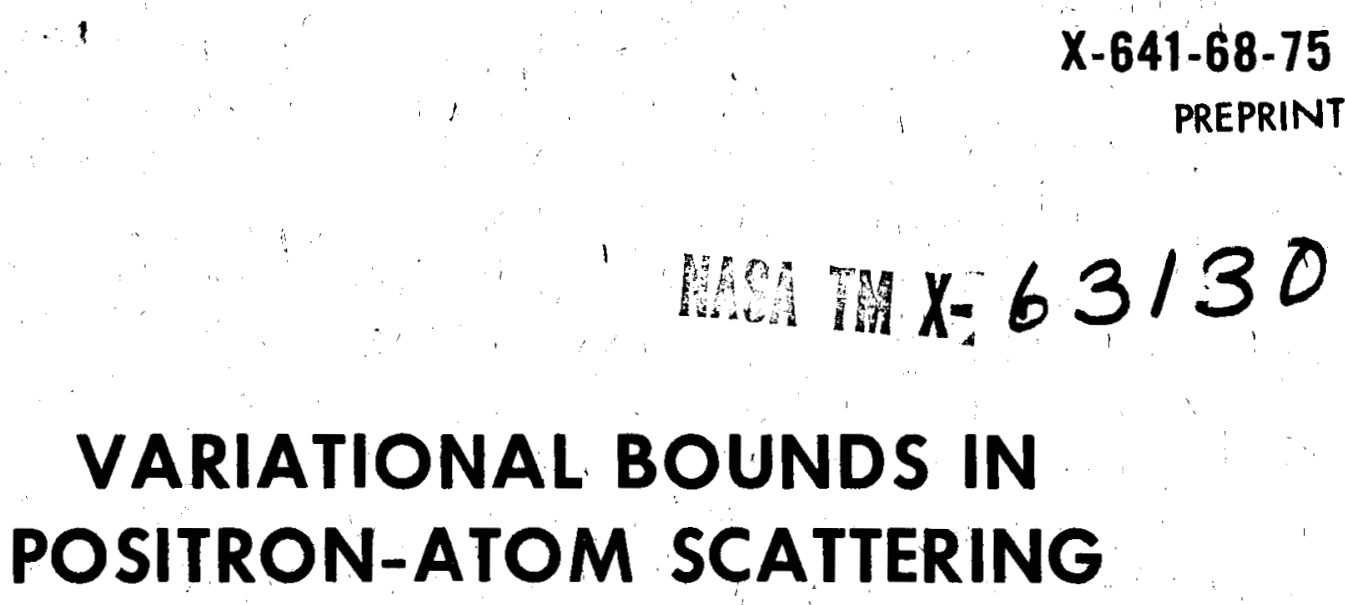

RICHARD J. DRACHMAN

GPO PRICE \$

CFSTI PRICE(S) \$

MARCH 1968

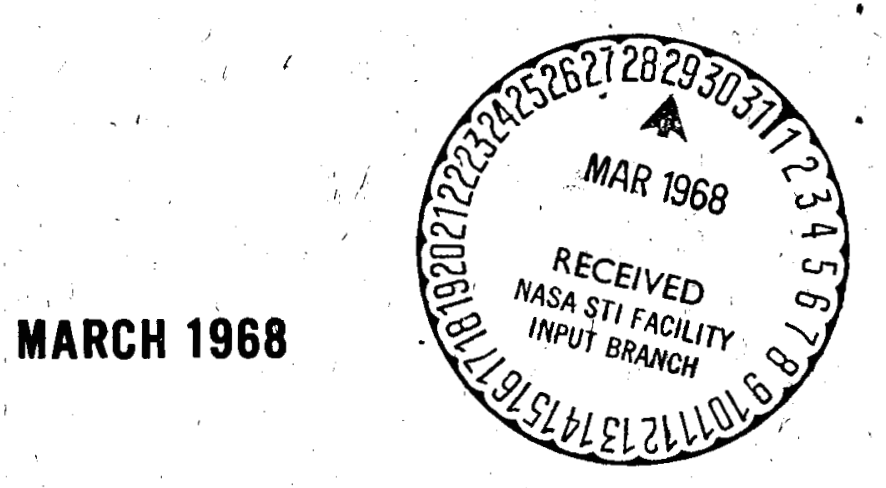

Hard copy $(\mathrm{HC})$

$3+8$

Microfiche (MF)

.65

ff 653 July 65

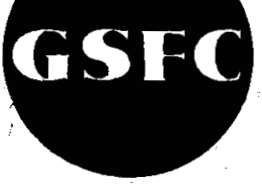

GODDARD SPACE FLIGHT CENTER

GREENBELT, MARYLAND

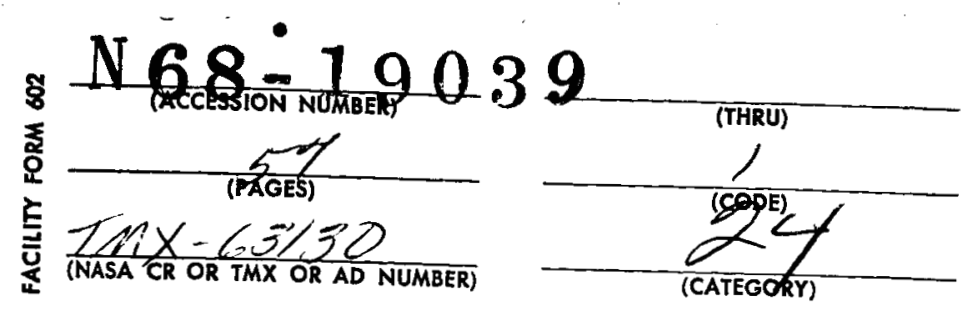




\title{
VARIATIONAL BOUNDS IN POSITRON-ATOM SCATTERING
}

\author{
Richard J. Drachman \\ NASA Laboratory for Theoretical Studies \\ Goddard Space Flight Center \\ Greenbelt, Maryland.
}

\begin{abstract}
We have previously applied the complete first-order adiabatic correlation function of Dalgarno and Lynn to the problem of low-energy elastic positron scattering by hydrogen and helium atoms. This approach is now extended to yield rigorous lower bounds on the scattering phase shifts in the case of hydrogen and "quasi-bounds" for helium. In addition, the positron annihilation rate in helium is re-evaluated, and lower values are now found, with the enhancement factor over the Dirac rate varying between 1.5 and 1.8. Some comparisons are made between the present method and other recent work.
\end{abstract}


I. Introduction

In two recent papers ${ }^{2}, 2$, the problem of low-energy elastic scattering of positrons from simple atoms (hydrogen and helium) has been treated in a modified adiabatic approximation. The method consisted in assuming that the optical potential ${ }^{3}$ is well represented by the posit1on-dependent second-order energy shift in the ground state of the target atom, due to the electric field of the positron ${ }^{4}$. Two novel features were introduced in these calculations. Firstly, the exact second-order potential ${ }^{5}$ was used in place of an approximate form ${ }^{4}$. Secondly, suppression of the short-range parts of the potential was accomplished by the use of a semi-empirical energy independent parameter, which served to reduce the spherical (monopole) distortion of the atom which would otherwise dominate as the positron approached the nucleus.

In the case of positron-hydrogen scattering ${ }^{1}, 90 \%$ suppression of the monopole was found to give essentially exact agreement with the definitive S-wave results of Schwartz ${ }^{6}$ from zero energy up to the positroniun formation threshold. Higher partial waves gave smaller phase-shifts than the best present estimates ${ }^{7}$, presumably due to the inclusion of insufficient amounts of virtual positronium in the wave function.

The positron-helium calculation ${ }^{2}$ was repeated for various values of monopole suppression, and suffered from the use of the shielded hydrogenic approximation for the atomic ground-state. But since no exact results exist its correctness cannot yet be evaluated ${ }^{8}$. The only existing experiment $^{9}$ measures the momentum transfer cross-section near the positronium 
formation threhold, and seems to disagree strongly.

The results for hydrogen ${ }^{2}$ seem to imply that the adiabatic wave function contains, to a considerable extent, the significant physics of low-energy positron scattering. Nevertheless, it is disturbing that a semi-empirical parameter is required, and also that no extremum principle or bound is contained in the results. The purpose of the present work ${ }^{10}$ is to use the adiabatic wave function in such a way as to obtain rigorous lower bounds to the phase silifts for $\mathrm{e}^{+}-\mathrm{H}$ scattering and "quasi-rigorous" lower bounds in the $\mathrm{e}^{+}-\mathrm{He}$ case.

In Section II, the Iower bound principle of Gailitis ${ }^{1}$ is adapted to the adiabatic type of trial function for $e^{+}-\mathrm{H}$ scattering. In Section III, a simple scale change gives the corresponding equations for $\mathrm{e}^{+}$- He scattering, and the $\mathrm{e}^{+}$annihilation rate in helium is also calculated. Results for these two cases are given and discussed in Section IV. In Section $V$ we clarify the relations between the present method and other recent work. Two Appendices contain details of the calculation. II. Formulation and Positron-Hydrogen Scattering.

We are interested here in obtaining approximate solutions to the positron-atom elastic scattering problem. The energy will be restricted so as to forbid any re-arrangement or inelastic processes, the lowest of which is real positronium formation, occurring above $6.8 \mathrm{eV}$ in hydrogen and $17.8 \mathrm{eV}$ in helium. To apply the lower bound principle of Gailitis ${ }^{1}$, it is sufficient in this energy range to use a trial scattering function of the form 
$-4-$

$$
\Psi_{k}(\Omega, x)=x_{k}(\underline{x}) \phi(\Omega)+\Phi(\Omega, x) .
$$

Here, $\underset{\sim}{x}$ is the position vector of the positron, $\underset{\sim}{\mathrm{r}}$ represents the atomic electrons, $\phi$ is the ground-state wave function of the target atom and $x$ is the scattering function for the positron of momentum $\underset{\sim}{\mathrm{k}}$, which must approach the correct asymptotic form as $\mathrm{x} \rightarrow \infty$. The distortion or polarization of the target by the incoming positron - the closed-channel part of the wave function - is approximately represented by $\Phi$, which satisfies the conditions ${ }^{12}$

$$
\int d^{3} \Omega \phi(\Omega) \Phi(\Omega, x)=0, \quad \text { for all } x
$$

(aa)

and

$$
\iint d^{3} x d^{3} \Omega[\Phi(\stackrel{\Omega}{\sim}, \underset{\sim}{x})]^{2}<\infty .
$$

$(2 \mathrm{~b})$ 
Then the two functions $X$ and $\Phi$ are to be determined. The resulting form of $x$ yields partial-wave phase shifts $\delta_{L}(k)$ which satisfy the inequality $\tan \delta_{L}(k) \leq \tan \delta_{L}(k)$ (exact), for all energies below any resonances that might occur.

The Hamiltonian for $\mathrm{e}^{+}-\mathrm{H}$ scattering is

$$
H=H_{r}+H_{x}+V \text {, }
$$

where $H_{1}=-\left[\nabla_{1}^{2}+2 / \Omega\right]$,

$$
H_{x}=-\nabla_{x}^{2}
$$

and

$$
V(\approx, \underset{\sim}{x})=2\left[\frac{1}{x}-\frac{1}{|x-\Omega|}\right],
$$

where we have set $h^{2} / 2 m_{e}=1, e^{2}=2$, with energies in Rydbergs and lengths in units of $a_{0}$, the Bohr radius. The correlation function is 


$$
\Phi(\Omega, x)=F(x) G(\Omega, x) \phi(\Omega)
$$

where $F$ is to contain short-range non-adiabatic modifications to the adiabatic correlation function $G$ which satisfies the first-order equation ${ }^{5}$

$$
\left[G, H_{\Omega}\right] \phi(\Omega)=[V-\langle v\rangle] \phi(\Omega)
$$

[Throughout this paper the bracket notation will represent expectation value in the ground state, ie

$$
\left.\langle V\rangle \equiv \int d^{3} \Omega \phi(\Omega) V(\Omega, x) \phi(r)\right]
$$

From Eq. (5), the function $G$ is determined to within an arbitrary additive function of $\mathbf{x}$, which may be chosen to make $\langle G\rangle$ vanish. With this choice, Eq. (4) is consistent with Eq. (2a).

If we use standard variational methods ${ }^{13}$ and allow $X$ and $F$ to undergo 
free, independent variation, we obtain the following two coupled equations :

$$
\begin{aligned}
& \langle[H-E][X+F G]\rangle=0, \\
& \langle G[H-E][X+F G]\rangle=0,
\end{aligned}
$$

where $E=E_{0}+k^{2}$ and $\left(H_{r}-E_{0}\right) \phi(r)=0$. Simple operations, including commutation of $\mathrm{H}_{\mathrm{r}}$ and $\mathrm{G}$ and use of $\mathrm{Eq}$. (5), yield the following coupled differential equations for $x$ and $F$ :

$$
\begin{aligned}
& {\left[\nabla^{2}+k^{2}-V_{1}\right] X=V_{2} F} \\
& {\left[N\left(\nabla^{2}+k^{2}\right)+V_{2}-V_{3}-W-V_{N} \frac{d}{d x}\right] F=V_{2} X .}
\end{aligned}
$$

The "potentials" appearing in Eq. (8) are defined as follows: 
$-8-$

$$
\begin{aligned}
& V_{p}(x)=\left\langle G^{p-1} \vee\right\rangle, \quad(p=1,2,3) ; \\
& W(x)=-\left\langle G \nabla_{x}^{2} G\right\rangle ; \\
& N(x)=\left\langle G^{2}\right\rangle ; \\
& V_{N}(x)=-\frac{d N}{d x} .
\end{aligned}
$$

(9)

Using the exact analytical form of $G$, given in elliptical coordinates by Dalgarno and Lynn $^{5}$, we have numerically evaluated ${ }^{14}$ all the functions defined in Eq. (9). The evaluation is outlined in Appendix A, and in Table I the potentials are tabulated.

The limiting forms of these potentials can be found analytically for small and large $x$, and are useful for starting the solutions of Eq. (8) and also to check their numerical evaluation. (See Appendix A) We obtain, for $x \rightarrow 0$

$$
\begin{aligned}
& V_{1} \approx 2\left[\frac{1}{x}-1\right] \\
& V_{2} \approx-1+0\left(x^{2}\right) \\
& V_{3} \approx \frac{3}{4} V_{1} \\
& N \approx \frac{3}{4}-\frac{5}{3} x^{2} \\
& V_{N} \approx \frac{10}{3} x \\
& W \approx 6[1-2 x],
\end{aligned}
$$


and for $\mathrm{x} \rightarrow \infty$

$$
\begin{aligned}
& V_{1} \text { and } W \rightarrow 0 \text { exponentially, } \\
& V_{2} \sim-\left[9 /\left(2 x^{4}\right)+15 / x^{6}+\right] \\
& V_{3} \sim-\left[213 /\left(2 x^{7}\right)+1773 / x^{9}+\right] \\
& N \sim\left[43 /\left(8 x^{4}\right)+107 /\left(8 x^{6}\right)+\right] \\
& \left.V_{N} \sim\left[43 /\left(2 x^{5}\right)+321 /\left(4 x^{7}\right)+\right]\right] .
\end{aligned}
$$

The solution of the coupled Eqs. (8) is outlined in Appendix B, and the results are discussed in Section IV. In Fig. I we have plotted the S-wave zero-energy solutions for the two functions $X$ and $F-X$, as well as the "suppression factor", $T(x)=F / X$. One can see the natural occurrence of the expected short-range suppression of $G$. An unexpected small enhancement also occurs for $\mathrm{x}>4$.

III. Positron-Helium Scattering

To extend the results of the preceding Section to the case of positron scattering from the ground state of helium, we interpret the correlation function of $\mathrm{Eq} .(4)$ as depending on two electronic coordinates, ie

$$
\Phi\left(\Omega_{1}, \Omega_{2}, \underset{\sim}{x}\right)=F(\underset{\sim}{x}) G\left(\underline{\sim}_{1}, \Omega_{2}, \underset{\sim}{x}\right) \phi\left(\Lambda_{1}, \Omega_{2}\right),
$$

where $\phi$ is the properly antisymmetric ground-state atomic wave function, 
$-10-$

and $\mathrm{G}$ is symmetric in the electronic coordinates.

The Hamiltonian for $e^{+}-$He scattering is

$$
H=H_{12}+H_{x}+V \text {, }
$$

where

$$
\begin{aligned}
& H_{12}=-\left[\nabla_{1}^{2}+\nabla_{2}^{2}+\frac{4}{\Omega_{1}}+\frac{4}{\Omega_{2}}\right]+\frac{2}{\left|\Omega_{1}-\Omega_{2}^{2}\right|}, \\
& H_{x}=-\nabla_{x}^{2},
\end{aligned}
$$

and

$$
V=V^{(1)}+V^{(2)} \text {, }
$$

where

$$
V^{(i)}=2\left[\frac{1}{x}-\frac{1}{\left|\Omega_{i}-x\right|}\right] .
$$

The assumption that $G$ is the first-order adiabatic correlation function requires $^{2}$

$$
\left[G, H_{12}\right] \phi\left(\Omega_{1}, \Omega_{2}\right)=[V-\langle V\rangle] \phi\left(\Omega_{1}, \Omega_{2}\right),
$$


where the bracket notation of Eq. (6) is extended to include integration over $\underset{\sim}{r_{1}}$ and $\underset{\sim}{r_{2}}$.

The formal equations to be solved are identical to Eq. (7) where $E=E_{0}+k^{2}$ and $\left(H_{12}-E_{0}\right) \phi\left(r_{1}, r_{2}\right)=0$. Using $E q$. (14) one obtains the following coupled equations:

$$
\left[\nabla^{2}+k^{2}-\langle v\rangle\right] X=\langle G V\rangle F,
$$

$$
\begin{array}{r}
{\left[\left\langle G^{2}\right\rangle\left(\nabla_{x}^{2}+k^{2}\right)+\langle G V\rangle-\left\langle G^{2} v\right\rangle+\left\langle G \nabla_{x}^{2} G\right\rangle\right.} \\
\left.+\nabla_{x}\left\langle G^{2}\right\rangle \cdot \nabla_{x}\right] F=\langle G v\rangle x .
\end{array}
$$

If it were possible to solve Eq. (14) exactIy, and to evaluate all the bracketed expressions in Eq. (15), the resulting phase shifts would satisfy the rigorous lower bound theorem. Since no exact solution for the helium atom ground state is known, one must use an approximate form for $\phi\left(r_{1}, r_{2}\right)$. We have used the simple, shielded hydrogenic form $\phi\left(r_{1}, r_{2}\right)=$ $\psi\left(r_{2}\right) \psi\left(r_{2}\right) S$, where $S$ is a singlet, antisymmetric spin function, and 


$$
\psi(\Omega)=\beta^{3 / 2} \pi^{-\frac{i}{2}} e^{-\beta \Omega}
$$

where $\beta$ is an effective charge. Since this function is not an exact eigenfunction of $\mathrm{H}_{12}$, the procedure leading to Eq. (15) is itself not really consistent. To the extent that these inconsistencies may be ignored however, the solutions of Eq. (15) will yield lower bounds to tan $\delta_{\mathrm{L}}$; we call these "quasi-bounds".

Using this form of $\phi$, it is consistent with Eq. (14) to assume independent distortion of the two electrons.

$$
G\left(\Omega_{1}, \Omega_{2}, x\right)=G\left(\Omega_{1}, x\right)+G\left(\Omega_{2}, x\right) \equiv G_{1}+G_{2} .
$$

Eq. (14) then simplifies to the form

$$
\begin{array}{r}
{\left[G_{i}, \nabla_{i}^{2}\right] \psi\left(\Omega_{i}\right)=\left[\left\langle V^{(i)}\right\rangle-V^{(i)}\right] \psi\left(\alpha_{i}\right),} \\
(i=1,2)
\end{array}
$$


where bracketed quantities now refer to single integrals, ie

$$
\left\langle V^{(i)}\right\rangle=\int d^{3} \Omega_{i} \psi\left(\Omega_{i}\right) V^{(i)} \psi\left(\Omega_{i}\right) .
$$

Again, one must set $\left\langle G_{i}\right\rangle=0$ to satisfy Eq. (2a). With this condition satisfied, one can immediately re-write Eq. (15) in terms of single integral quantities:

$$
\begin{aligned}
& {\left[\nabla^{2}+k^{2}-2 V_{1}\right] X=2 V_{2}^{\prime} F_{1}} \\
& {\left[N\left(\nabla^{2}+k^{2}-V_{1}\right)+V_{2}-V_{3}-W-V_{N} \frac{d}{d x}\right] F=V_{2} X}
\end{aligned}
$$

All the "potentials" used in Eq. (20) have the formal definitions of Eq. (9), but with $\phi(r)$ replaced by $\psi(r)$. The factors of 2 appearing in $\mathrm{Eq}$. (20a) are due to the superimposed effects of the two electrons. In Eq. (20b), there is an additional term, $-\mathrm{NV}_{1}$, which did not appear for hydrogen, Eq. (8b). This comes from the third-order term in Eq. (15b), 


$$
\begin{aligned}
\left\langle G^{2} V\right\rangle= & \left\langle\left(G_{1}+G_{2}\right)^{2}\left(V^{(1)}+V^{(2)}\right)\right\rangle \\
= & \left\langle G_{1}^{2} V^{(1)}\right\rangle+\left\langle G_{2}^{2} V^{(2)}\right\rangle \\
& \left.+\left\langle G_{1}^{2}\right\rangle\left\langle V^{(2)}\right\rangle+\left\langle G_{2}^{2}\right\rangle\left\langle V^{(1)}\right\rangle\right\rangle \\
& +2\left\langle G_{1}\right\rangle\left\langle G_{2} V^{(2)}\right\rangle+2\left\langle G_{2}\right\rangle\left\langle G_{1} V^{(1)}\right\rangle
\end{aligned}
$$

The last two terms vanish, and the net result is

$$
\left\langle G^{2} V\right\rangle=2\left[V_{3}+N V_{1}\right]
$$

It has been noted ${ }^{2}$ that a simple change of scale converts Eq. (18) into its hydrogen equivalent, Eq. (5). Specifically, let $\underset{\sim}{\mathrm{y}}=\underset{\sim}{\mathrm{r}}$ and $\stackrel{\mathrm{v}}{\sim}=\underset{\sim}{ } \cdot$ Then Eq. (18) becomes 
$-15-$

$$
\left[\beta G, \nabla_{y}^{2}\right] e^{-y}=\left[\frac{2}{|y-v|}-\frac{2}{v}-\bar{V}_{1}(v)\right] e^{-y}
$$

(23)

where

$$
\nabla_{1}(v)=\frac{2}{\pi} \int d^{3} y e^{-2 y}\left[\frac{1}{v}-\frac{i}{\mid y-v i}\right] .
$$

One sees immediately from Eqs. (23) and (5) that

$$
\beta G_{\text {He }}(\stackrel{\sim}{\sim}, \underset{\sim}{x})=G_{H}(\underset{\sim}{y}, \underset{\sim}{v}) \text {. }
$$

Also, since $V_{\mathrm{He}}(\underset{\sim}{\sim}, \underset{\sim}{\mathrm{x}})=\beta V_{\mathrm{H}}(\underset{\sim}{\mathrm{y}}, \underset{\sim}{\mathrm{v}})$, and $\psi_{\mathrm{He}}^{2}(r) \mathrm{d}_{\sim}^{3} \mathrm{\sim}=\phi_{\mathrm{H}}^{2}(\mathrm{y}) \mathrm{d}^{3} \underset{\sim}{\mathrm{y}}$ one can relate all the helium potentials of Eq. (20) to the hydrogen potentials of Eq. (8) as follows:

$$
\begin{aligned}
& V_{p}(x) \rightarrow \beta^{2-p} V_{p}(v), \quad(p=1,2,3) \\
& N(x) \rightarrow \beta^{-2} N(v), \\
& V_{N}(x) \rightarrow \beta^{-1} V_{N}(v), \\
& W(x) \rightarrow W(v) .
\end{aligned}
$$

(25) 
The simplest way to make use of these scaling laws is to change variables throughout Eq. (20):

$$
\begin{gathered}
{\left[\nabla^{2}+k^{i^{2}}-\frac{2}{\beta} V_{1}\right] X=\frac{2}{\beta^{2}} V_{2} F,} \\
{\left[N\left(\nabla^{2}+k^{\prime 2}-\frac{1}{\beta} V_{1}\right)+V_{2}-\frac{1}{\beta} V_{3}-W-V_{N} \frac{d}{d v}\right] F} \\
=V_{2} X .
\end{gathered}
$$

Here $k^{\prime}=k / \beta, \nabla^{2} \equiv \nabla_{\mathrm{v}}^{2}$, and all the potentials are the hydrogen functions in terms of $\mathrm{v}$. These equations can be treated just like those appearing in the hydrogen problem, as discussed in Appendix B, and the results are discussed in Section IV. As indicated previously ${ }^{2}$, the value $\beta=\frac{27}{16}$ which minimizes the energy expectation value gives an unsatisfactory value (1.11) for the polarizability of helium. This quantity appears in the present theory as $\lim _{x \rightarrow \infty}\left[2 x^{4} V_{2}(x)\right]=9 B^{-4}$. With only a slight change in the energy, one can adjust the polarizability to the experimental value ${ }^{2}$ of 1.376 , by setting $\beta=1.5992$. This value has been used throughout the present work.

We have previousl $y^{15}$ used the non-variational $e^{+}-$He wave functions to calculate $\mathrm{z}_{\mathrm{k}}$, the number of atomic electrons which are effective in 
$-17-$

annihilating a positron of momentum k. (For planewave positrons and omitting atomic distortion, $Z_{k}=2$ ). In terms of our type of wave function,

$$
\begin{aligned}
\frac{1}{2} Z_{k} & =\int d^{3} \Omega \int d^{3} x\left|\psi_{k}(\Omega, x, x)\right|^{2} \\
& =\int d^{3} x \psi^{2}(x)\left[|x+F G(x, x)|^{2}+|F|^{2}\left\langle G^{2}\right\rangle\right] .
\end{aligned}
$$

(27)

Following Ref. 15, we assume that only the $L=0$ scattering function differs significantly from its planewave form, and that for $I>0$ the ratio of closed-channel to open-channel functions is well approximated by the zero-energy suppression function T. With these approximations, one can write

$$
\begin{aligned}
\frac{1}{2} Z_{k}= & \left(\frac{2 \beta}{k}\right)^{2} \int_{0}^{\infty} d v e^{-2 v}\left[\left(v_{0}+g_{0} G(v, w)\right)^{2}+g_{0}^{2} N(v)\right] \\
& +4 \int_{0}^{\infty} d v e^{-2 i} v^{-2} M(v)\left[1-\gamma_{0}^{2}\left(\frac{k i r}{\beta}\right)\right],
\end{aligned}
$$


where

$$
M\left(v^{-}\right)=[1+T G(u, w)]^{2}+T^{2} N\left(i^{-}\right)
$$

Here $\mathrm{v}=\beta \mathrm{x}, \mathrm{U}_{0}$ and $\mathrm{g}_{\mathrm{O}}$ are the $\mathrm{S}$-wave parts of $x$ and $\mathrm{F}$ (see Eq. BI). The correlation functions used here $[G(\underset{\sim}{v}, \underset{\sim}{v})$ and $N(v)]$ are scaled appropriately for helium. At $k=0$ the second integral in Eq. (28) vanishes since no higher $L$-values contribute. For $k \neq 0$ the assumptions made here should be quite good; in Ref. I5 we have estimated that an error of less than $2 \%$ is incurred in the values of $\mathrm{Z}_{\mathrm{k}}$. In Table II the results are given for values of the energy up to the positroniun threshold, in comparison with the larger values of $\mathrm{Z}_{\mathrm{k}}$ previously obtained ${ }^{15}$. The difference between the present results and those obtained previously is traceable partly to the fact that the present calculation gives less attraction than did the non-variational method ${ }^{15}$, and hence the positron is pulled inward to a smaller degree. A larger effect is the difference between the "correlation function" $\mathrm{M}$ defined in Eq. (28) and the monopole - suppressed function $\mathrm{Y}$ used in Ref. (15). These two functions are compared in Table III, and one can see that, except at very small distances from the nucleus, $M<Y$. To examine these two effects quantitatively, we have re-computed $\frac{l}{2} z_{k}$ at zero energy using both $M$ and $Y$, as well as both variational and non-variational scattering functions. By far the largest effect is produced by replacing $M$ by $Y$, outweighing the effect of interchanging the two forms of $U$ by a factor of 3 . Since neither $M$ nor $Y$ is exact, this result constitutes a warning against taking the annihilation results too seriously. It is interesting, however, that the present calculation agrees fairly well at zero energy with the experi- 
mental result of Falk et al ${ }^{16}$ for thermal positrons of $z_{k}=3.78 \pm 0.17$. IV. Scattering Results and Discussion

The $\mathrm{e}^{+}$- H S-wave scattering phase shifts $\delta_{0}$ were computed numerically as discussed in Appendix $B$, where the method of treating the asymptotic behavior of the closed-channel is discussed. This involves inward integration from a point $x_{0}$ in the asymptotic region to an intermediate point $\bar{x}$. In Table IV the sensitivity of $\tan \delta_{0}$ to the choice of $x_{0}$ and $\bar{x}$ is shown for low and high-energy positrons, confirming the stability of the numerical solutions.

In Table $\mathrm{V}$ the $\mathrm{e}^{+}-\mathrm{H} \mathrm{S}$-wave phase shifts (and, at $\mathrm{k}=0$, the scattering length) are shown, along with the results of Schwartz ${ }^{6}$. To assess the quality of the present work, we compute the quantity $\Delta$, the difference between $\tan \delta_{0}$ and the value of $\tan \delta_{0}$ obtained with an undistorted atomic wave function. This "Hartree" result is also given in Table V. The figure of merit $Q(k) \equiv \Delta($ variational $) / \Delta$ (Schwartz), measures how much of the distortion or polarization has been accounted for by our method. It is most interesting that $Q \approx 90 \%$, almost independent of energy. The lower bound results of Hahn and Spruch $^{17}$ have somewhat lower Q-values, as shown in the Table. We consider it a success of the present method that the results compare well with the many-parameter, many- $l$ variational results of Ref. 17. Their method has the considerable advantage, however, of being capable of systematic improvement, by the addition of more terms. The results can also be convincingly extrapolated, and give good agreement with those of Schwartz ${ }^{6}$.

In Table VI the S-wave scattering results for $\mathrm{e}^{+}$- He are presented. 
Along with the "quasi-rigorous" Iower bounds obtained here, we show the "Hartree" results, obtained from a two-term analytic form² approximating the helium ground-state potential, and the non-variational results ${ }^{2}$. Since no definitive calculation exists in this case, we have computed the figure of merit $Q$ assuming that $\delta_{\mathrm{NV}}$ is exact. These Q-values are smaller than those obtained for hydrogen, but are also nearly constant. It is not possible to make any further statements about the "correct" values for the $S$-wave $e^{+}$- He phase shifts from the comparison of the quasi-variational and non-variational results.

The possibility that a bound $e^{+}-e^{-}-$P exists has been eliminated by an extensive variational calculation 18 which showed that for a "positron" of mass m $>2.625$ such a bound system does occur. As a further test of the present method, we have calculated this critical mass by looking for the appearance of a node in the S-wave zero-energy scattering function, corresponding to a scattering lenght $a= \pm \infty$. One modifies Eq. (8) by dividing all terms involving $\nabla_{x}^{2}$ by the "positron" mass $m$. This is equivalent to multiplying the potentials $\mathrm{V}_{1}, \mathrm{~V}_{2}, \mathrm{~V}_{3}$ by $\mathrm{m}$, and adjusting the asymptotic conditions in an obvious way. We find the critical mass to be 2.79 , and since our method gives an upper bound this agrees with the result of Ref. 18 .

The present method is not expected to be as good for $\mathrm{L}>0$ as for $I=0$, since even the non-variational results ${ }^{1}$ underestimate the $\mathrm{P}$ - and D.-wave phase-shifts at the higher energies. This defect seems to be associated with a lack of sufficient virtual positronium in the wave function. Support for this is the success of Bransden's P-wave results ${ }^{19}$ 
at high energy. More recent results ${ }^{20}$ indicate that just above the threshold for positronium formation, $\mathrm{P}$-waves dominate over $\mathrm{S}$-waves in the pick-up process, implying that below threshold neglect of positronium is less serious for $\mathrm{S}$-waves. In any case, we show our $\mathrm{L}=\mathrm{I}, \mathrm{e}^{+}-\mathrm{H}$ results in Table VII, where they are compared with the Iower-bound and extrapolated results of Kleinman et al ${ }^{21}$.

V. Comparison with Other Methods

We will now describe briefly some other methods which resemble the present work, hoping that some clarification will result. The field of low-energy positron scattering suffers from some lack of precision in terminology and some overlapping of approximations, which may make it difficult to evaluate the work reported here without a description of the competing methods. The following short review is not complete, but it may aid the orientation of the reader.

1. The non-variational (polarized orbital) method ${ }^{1}$ uses the form of scattering function given in Eqs. (1) and (4) with the additional restriction $F(\underset{\sim}{x})=x(\underset{\sim}{x})$. Since it is non-variational, however, the single unknown function $x$ is determined by simply projecting the Schrodinger equation onto the open-channel part of $\Psi$ :

$$
\langle[H-E][1+G]\rangle X=C .
$$


This leads to the differential equation

$$
\left[\nabla^{2}+k^{2}\right] X-\left[v_{1}+v_{2}\right] X=0,
$$

whose solutions gives the phase shifts. In the absence of an applicable lower bound principle it is difficult to gauge the error in the phase shifts obtained from Eq. (30).

2. The same polarized orbital wave function can be employed variationally. The same procedure which leads to Eq. (7) when $X$ and $F$ are independently varied, leads now to

$\langle[1+G][H-E][1+G]\rangle X=0$

since $F \equiv X$. This gives the differential equation

$$
\left[\nabla^{2}+k^{2}\right] x-[1+N]^{-1}\left[V_{1}+V_{2}+V_{3}+W+V_{N} \frac{d}{d x}\right] x=0,
$$




\section{$-23-$}

whose solutions provide approximate phase shifts which are lower bounds to the exact ones. Unfortunately, these approximate phase shifts are very low $(a \simeq-1.0)$, since the large, short-range repulsion represented by $\mathrm{W}$, and the reduction in $V_{2}$ caused by the factor $(I+N)^{-1}$ more than overcome the attraction added by $V_{3}$. It is Iikely that removing some or all of the monopole part of $G$, as was done non-variationally in Ref. 1, would improve the results considerable. If $G$ were replaced by $G-(I-\alpha) G_{0}$, the potentials appearing in Eq. (32) would be modified as follows:

$$
\begin{aligned}
& N \rightarrow N-\left(1-\alpha^{2}\right) N_{0} \\
& W \rightarrow W-\left(1-\alpha^{2}\right) W_{0} \\
& V_{N} \rightarrow V_{N}-\left(1-\alpha^{2}\right) V_{N O} \\
& V_{1} \rightarrow V_{1} \\
& V_{2} \rightarrow V_{2}-(1-\alpha)^{2} V_{20} \\
& V_{3} \rightarrow V_{3}-\left(1-\alpha^{2}\right) V_{30}-2(1-\alpha) P
\end{aligned}
$$

where, for example, $N_{0} \equiv\left\langle G_{0}^{2}\right\rangle$, and $P \equiv\left\langle G_{0}\left(G-G_{0}\right)\left(V-V_{0}\right)\right\rangle$.

In Eq. (33), the parameter $\alpha$ represents the fraction of adiabatic monopole distortion being retained; $\alpha=0$ is what we have called "full monopole suppression". The best value of $\alpha$, for each $L$ and $k$, is to be determined variationally. 
Eq. (32) is integrated for several values of $\alpha$, and the value which maximizes the phase shifts is retained. The monopole terms (with subscript 0 ) are easily evaluated in spherical coordinates, while the remaining terms are know in elliptical coordinates, except for the last term in $V_{3}$. The quantity $P$ is difficult to compute in either set of co-ordinates, but it might be approximated using the multipole expansions for $G$ and $V$. This method has not been used up to the present time.

3. Callaway et al ${ }^{22}$ have made some modifications to Eq. (32) and arrive at another optical potential which they call the extended polarization potential. Although the results obtained are poor, the new potential has considerable physical interest.

To derive the result of Ref. (22), one first neglects all terms which are formally of higher than second order in the perturbation V. This eliminates from Eq. (32) all reference to $\mathrm{N}$ and $\mathrm{V}_{3}$, and leaves us with Eq. (30) modified by the addition of the two second-order non-adiabatic potertials $W+V_{N} \frac{d}{d x}$. Further arguments in Ref. (22) are used to justify a certain normalization of the scattering function, but in essence one next introduces a new function $\bar{x}$ by the definition

$$
x=\left(1-\frac{1}{2} N\right) \bar{x}
$$


and a differential equation for $\bar{x}$ is derived by direct substitution. Again neglecting terms higher than second order, one obtains the equation

$$
\left[\nabla^{2}+k^{2}\right] \bar{x}-\left[V_{1}+V_{2}+V_{D}\right] \bar{x}=0,
$$

where $\mathrm{V}_{0}$ is the "distortion potential" $<\left(\nabla_{\mathrm{X}} \mathrm{G}\right)^{2}>$ appearing in Appendix A. From Eqs. (A4) and (10) we find that $V_{D}=1$ at $x=0$. Thus, as Callaway et al ${ }^{22}$ have emphasized, the distortion potential cancels the polarization potential $V_{2}$ at the origin, and hence its inclusion seems to serve the same purpose as does monopole suppression ${ }^{1}$. Unfortunately $V_{D}$ is fairly long range, decreasing like $x^{-6}$ for large $x$, while $\mathrm{V}_{20}$ exponentially decreases. The equivalence of these two ways of suppressing excessive attraction is limited to small $\mathrm{x}$, and consequently Eq. (35) gives far too much repulsion; the scattering length is ${ }^{22} \mathrm{a}=-0.783$, even worse than the result of Eq. (32).

4. We have previously reported ${ }^{10^{a}}$ a method of obtaining lower bounds to the phase shifts, which is simpler than that of the present work and not much different in its results. In essence it is a hybrid between the present method, which independently varies two functions and obtains coupled differential equations, and the method of Eq. (3I) which varies 
only one function.

In this method, one again uses a trial function of the type represented by Eqs. (1) and (4), with

$$
F(\underline{x})=X_{k}(\underline{x}) T(x),
$$

where $T(x)$ is some function which approaches unity for large $\mathrm{x}$ and decreases for small $\mathrm{x}$. It is to contain adjustable constants ${ }^{23}$, and is designed to suppress the short-range correlation. Variation on $x$ leads to the following equation, analogous to Eq. (3I):

$$
\langle[1+T G][H-E][1+T G]\rangle X=C .
$$

The optical potential of Eq. (32) is modified, and the lower bound phase shifts are solutions of the differential equation 


$$
\begin{gathered}
{\left[\nabla^{2}+k^{2}\right] X-\left[1+T^{2} N\right]^{-1}\left[V_{1}^{\prime}+\left(2 T-T^{2}\right) V_{2}\right.} \\
+T^{2}\left(V_{3}+W\right)+\left(T^{2} V_{N}-2 T^{\prime} N\right) \frac{d}{d x} \\
\left.+\left(T \nabla^{2} T\right) N+T T^{\prime} V_{N}\right] X=0,
\end{gathered}
$$

which is seen to reduce to Eq. (32) if $T=1$. For $k=0$, $L=0$ scattering, the optimum form of $T$ would be that shown in Fig. $I$, where $T=F / X$, while $F$ and $X$ were numerically obtained. For $k \neq 0$, however, $x$ and $F$ both are oscillatory but their zeroes do not generally coincide, and one must use a smoothed approximate form for $\mathrm{T}$, as has been done for the annihilation problem in section III. It is also possible to combine the monopole suppression method with the present method. In that case, the constant $\alpha$ would measure the amount of monopole correlation retained, while the function $T$ would be capable of making adjustments in the remaining parts of the correlation function.

5. Two interesting modifications of the close-coupling approximation have been introduced recently. They are quite close in approach to the present coupled-equation method.

a. Damburg and Karule $e^{24}$, noticing that the close-coupling 
method fails to give the correct asymptotic dipole polarizability, have proposed a trial function which is equivalent to our Eqs. (I) and (4), with a different form for $G$ :

$$
\begin{array}{r}
C_{D K}(\Omega, x)=\left(\Omega+\frac{1}{2} \Omega^{2}\right) P_{1}(\cos n), \\
(\cos n=\hat{\imath} \cdot \hat{x}) .
\end{array}
$$

The motivation for this choice is clear: comparing Eq. (39) with Eq. (A8), one sees that $G_{D K}$ correctly describes the asymptotic form of the adiabatic correlation function, as far as the coordinates of the electron $(r, \eta)$ are concerned, and since $F(\underset{\sim}{x})$ can be freely varied, the solutions of the resulting coupled equations will be identical, for large $\mathrm{x}$, to those of Eq. (8). Eq. (39) is separable in $r, \eta, x$, however, and hence does not give the same short-range dipole correlation as does as does the Dalgarno-Lynn function G. In addition, each mutipole term in the correlation function would require another coupled equation (as is the case for ordinary close-coupling). In return one gains enormously in the simplicity of the potentials which must be used; all the potentials in this method can be evaluated analytically, while some reduce trivially to constants. The method is promising, but no numerical 
results are available yet.

b. Perkins ${ }^{25}$ has made a modification starting from

a conventional close-coupling expansion containing

Is - $2 p-3 d$ states. Noting that, since only one

term of each angular symmetry is retained, the ortho-

gonality of closed and open channels [Eq. (2a)] is

assured, he has modified the forms of the $2 p$ and $3 d$

functions as follows

$$
\begin{aligned}
& \phi_{2 p^{\prime}}=\Omega e^{-\delta \Omega} P_{1}, \\
& \phi_{3 d^{\prime}}=\Omega^{2} e^{-\eta \Omega} P_{2} .
\end{aligned}
$$

The ususal coupled differential equations of closecoupling are solved numerically, but now two additional non-linear parameters, $\delta$ and $\eta$, are adjusted to maximize the phase shifts. The results are much better than the corresponding unmodified close-coupling results, although the exact asymptotic polarizability is not achievable. The method can be extended to include higher $\ell$-values with the usual difficulty that more coupled equations are then required. Without generalizing the basis functions considerably, one would not expect the procedure to converge toward the exact wave function, but the lower bound theorem holds and offers the usual estimate of quality. 
6. An interesting general formation was recently presented ${ }^{26}$, which uses numerical solutions of the two-center problem in the adiabatic approximation, and which may prove applicable to positron scattering. Similarities with our method include generalizations of our $V_{N}$ and $W$. The binding energy of the $e^{+}-e^{-}-e^{+}$molecule was computed as an example, but no scattering results were given.

\section{Acknowledgments}

The numerical caluclations reported here were programmed by Edward Monasterski for the IBM 360 electronic computer at the Goddard Laboratory for Theoretical Studies. Critical and constructive discussions with Dr. Aaron Temkin are gratefully acknowledged. A correspondence with Dr. R. W. LaBahn pointed out some significant errors in evaluation of the important function W. Dr. M. Inokuti first brought the thorough work of Ref. (18) to the author's attention. Helpful discussions with Dr. J. F. Perkins, Dr. R. Damburg, and Professor B. H. Bransden were also appreciated. 
$-31-$

Appendix A. Evaluation of the Potentials

The analytic solution of Eq. (5) was given by Dalgarno and Lynn ${ }^{5}$ and has the form

$$
\begin{aligned}
G= & A(x)[\lambda+\mu]+\left(1+\frac{2 A}{x}\right) \ln (1+\lambda) \\
& +\left(1+\frac{1}{x}\right) Q(x, \mu)+\bar{G}(x),
\end{aligned}
$$

where $\quad A(x)=\frac{1}{2}\left[(x+1) e^{-2 x}-1\right]$,

$$
\begin{aligned}
Q(x, \mu)=E_{i}(x[\mu-1]) & -\ln (1-\mu) \\
& -e^{-2 x}\left[E_{i}(x[\mu+1])-\ln (1+\mu)\right],
\end{aligned}
$$

and $E_{i}(-z)=-\int_{z}^{\infty} d y e^{-y} / y$. In terms of these elliptical coordinates, $r=\frac{1}{2} x(\lambda+\mu)$. and $\cos \eta=(I+\lambda \mu) /(\lambda+\mu)$, where $\eta$ is the angle between $\underset{\sim}{x}$ and $\underset{\sim}{\sim}$ The potential $\mathrm{V}=\frac{2}{\mathrm{x}}\left[1+\frac{2}{\mu-\lambda}\right]$, the bracket notation takes the form

$$
\langle f(x, \lambda, \mu)\rangle=\frac{1}{4} x^{3} \int_{1}^{\infty} d \lambda \int_{-1}^{1} d \mu e^{-x(\lambda+\mu)}\left(\lambda^{2}-\mu^{2}\right) f(x, \lambda, \mu)
$$

$(\mathrm{A} 2)$ 
and we may determine $\bar{G}(x)$ by requiring $\langle\bar{G}\rangle=0$, obtaining

$$
\bar{G}(x)=\frac{2}{x}\left[\ln 2-E_{i}(-2 x)\right]+e^{-2 x}\left[2\left(1+\frac{1}{x}\right) \ln \gamma x+x-\frac{1}{2}\right],
$$

where $\ln _{\gamma}=0.5772 \ldots$. , Euler's constant. The solubility of Eq. (5) depended on its additive separability in elliptical coordinates. For the same reason, it is possible to evaluate most of the potentials defined in Eq. (9) in terms of one-dimensional integrals. Since, however, the important function $\mathrm{W}$ cannot be reduced in this way, we have numerically evaluated all the potentials uniformly by the use of Gaussian quadrature in two dimensions. We have confirmed the accuracy of this procedure in three ways: We have evaluated $\langle\mathrm{V}\rangle,\langle\mathrm{G}\rangle$, and $\langle\mathrm{GV}\rangle$ in elliptical coordinates, using our numerical method, and compared the results with the known values, obtaining excellent agreement. In particular, $\langle G\rangle$ never differs from zero by more than about $10^{-7}$, and we feel that this figure measures the accuracy of the two-dimensional integrals.

The two non-adiabatic terms, $V_{\mathrm{N}}$ and $\mathrm{W}$, present additional problems. To evaluate $W$ directly would involve the operation $\nabla_{x}^{2}$, which would give a very intricate analytic expression. Instead, we have re-written the expression for $\mathrm{W}$ in the form 


$$
W=V_{D}-\frac{1}{2} \nabla^{2} N=V_{D}+\left(\frac{1}{x}+\frac{1}{2} \frac{d}{d x}\right) V_{N}
$$

where $V_{D} \equiv\left\langle(\underset{\sim X}{\nabla} G)^{2}\right\rangle$ is the "distortion potential" employed in Ref. 22, and discussed here in Section $V$. The function $V_{D}$ involves both $x$ and $\mathrm{n}$ components of the gradient (with $\mathrm{r}$ held fixed), while $\mathrm{V}_{\mathrm{N}}$ only requires the $\mathrm{x}$ component. Both components were found analytically and inserted into the numerical double integration, while the first derivative of $V_{N}$ needed in Eq. (A4) was gotten by numerical differentiation of $V_{N}$. All three terms in Eq. (A4) have asymptotic inverse-power behavior, with $\mathrm{x}^{-6}$ as the leading term; these all cancel leaving short-range (exponential) terms dominant.

In Section III we require the value of $G(\underset{\sim}{x}, \underset{\sim}{x})$, ie, $G(\underset{\sim}{r}, \underset{\sim}{x})$ evaluated at $\underset{\sim}{r}=\underset{\sim}{x}$. In elliptical coordinates this relation becomes $\lambda=\mu=1$, since then $r=x$ and $\cos \eta=I$. Care is required in evaluating $Q(x, 1)$, since its first two terms are singular. Letting $\mu=I-\epsilon$ and expanding the exponential integral about $\epsilon=0$, we have 
$-34-$

$$
C(x, 1-\epsilon) \approx \ln (\gamma \in x)-\epsilon x-\ln \epsilon-e^{-2 x}\left[E_{i}(2 x)-\ln 2\right],
$$

(AF)

and the limit $\epsilon \rightarrow \circ$ can be taken. The final result is

$$
\begin{aligned}
G\left(x, \frac{x}{2}\right) & =\left(1+\frac{1}{x}\right)\left[\ln (2 \gamma x)+e^{-2 x}\left(2 \ln [2 \gamma x]-E_{i}[2 x]\right)\right] \\
& -1-\frac{2}{x} E_{i}(-2 x)+e^{-2 x}\left(\frac{1}{2}+2 x\right)
\end{aligned}
$$

This is the function which, properly scaled, is used in the $e^{+}-\mathrm{He}$ annihilation and forms the basis for Table III.

To obtain the long-range asymptotic forms shown in Eq. (II), we expand $\mathrm{V}$ in Legendre polynomials (for $\mathrm{x}>\mathrm{r}$ ):

$$
V^{\prime}(\Omega, x) \sim-\frac{2}{x} \sum_{l=1}^{\infty}\left(\frac{r}{x}\right)^{l} P_{l}(\cos \eta)
$$


$-35-$

Then a particular solution of Eq. (5) is ( for $x>r$ )

$$
G=\frac{1}{x} \sum_{l=1}^{\infty}\left(\frac{r}{x}\right)^{l}\left(\frac{1}{l}+\frac{r}{l+1}\right) P_{l}(\cos n),
$$

which is the correct asymptotic form of G. By inserting these expressions into the definitions, Eq. (9), one can carry out the integrations in spherical coordinates and obtain the leading terms, (in $\mathrm{x}^{-1}$ ) for each potential. Since $V$ has no long-range $\ell=0$ term, $V_{1}$ is short-ranged. To show that $W$ is also, one notes that $\nabla_{x}^{2} x^{-(l+1)_{P}} \ell_{\ell}(\cos \eta)=0$, for $\ell>0$. To illustrate, let us compute the asymptotic form of $\mathrm{N}$ :

$$
N \equiv\left\langle G^{2}\right\rangle \sim \frac{2}{x^{4}} \int_{0}^{\infty} d r r^{2} e^{-2 r} r^{2}\left(1+\frac{r}{2}\right)^{2} \int_{-1}^{1} d(\cos \eta)\left[P_{1}(\cos \eta)\right]^{2},
$$

or $N \sim 43 /\left(8 x^{4}\right)$

(AID) 
The expansion can be carried to any desired order in $l$, and represents an asymptotic expansion, since we have set the upper limit of the integral equal to $\infty$ rather than $x$. Care is required in evaluating the asymptotic form of $V_{3}$ since more than one value of $\&$ contributes to the same order in $\mathrm{x}^{-1}$, because three Legendre polynomials appear.

The short-range behavior of the potentials (Eq. 10) is due to the small- $\ell$ parts of the distortion function $G$. In Ref. I. the $l=0$ solution of Eq. (5) is exhibited. Near $x=0$ it becomes

$$
G_{0} \approx \frac{3}{2}-\Omega+\frac{2}{3} x^{2}(1-x)\left[\ln (2 \gamma r)+r-\frac{1}{2 r}-\frac{5}{2}\right] \text {, }
$$

and

$$
\nabla_{x}^{2} G_{c} \approx 4(1-2 x)\left[\ln (2 r \mu)+r-\frac{1}{2 r}-\frac{5}{2}\right] .
$$

Since, for small $x$, all the integrals have limits of 0 and $\infty$, one finds 


$$
\begin{aligned}
& N_{0} \equiv\left\langle G_{0}^{2}\right\rangle \approx \frac{3}{4}-2 x^{2}, \\
& W_{0} \equiv-\left\langle G_{0} \nabla_{x}^{2} G_{0}\right\rangle \approx 6[1-2 x], \\
& V_{N 0} \equiv-\frac{d}{d x} N_{0} \approx 4 x .
\end{aligned}
$$

The constant terms in Eq. (Al3) are unaffected by the neglect of higher $\ell$-values, but the second term in $N$ and, hence, the leading term in $V_{N}$, is modified by the $l=1$ part of the expansion. One finds

$$
G_{1} \approx P_{1}(\cos n)\left[x+C\left(x^{3}\right)\right],
$$

and, $N_{1} \equiv\left\langle G_{1}^{2}\right\rangle \simeq \frac{1}{3} x^{2}$. No higher terms contribute to order $x^{2}$, and we obtain the results given in Eq. (10). The $l=0$ and $l=1$ parts of $\mathrm{V}_{\mathrm{D}}$ can now be found from Eq. (A4). The result is $\mathrm{V}_{\mathrm{DQ}} \approx 0$ and $\mathrm{V}_{\mathrm{DI}} \approx I$ near $x=0$, and no higher terms contribute. 
Appendix B. Solution of the Scattering Equations

The numerical integration of Eq. (8), in partial wave decomposition, would be straightforward were it not for difficulties connected with the asymptotic form of the closed-channel part of the trial function, Eq. (I). The problem is very similar to those arising in the close-coupling method with closed channels, and our treatment follows the review by Burke and Smith $^{27}$ fairly closely.

Since we are dealing here with a set of two coupled second-order differential equations, four linearly independent solutions can be found, with each solution consisting of a pair of functions $(X, F)$. We will see later that only two of these solutions are sufficiently regular at $x=0$ to be admissable. The general solution of Eq. (8) is thus a linear combination of these two solutions, and our problem is to determine such a combination which is sufficiently regular as $x \rightarrow \infty$. Making partial wave expansions as follows

$$
\begin{aligned}
& X(\underline{x})=\sum_{L=0}^{\infty} x^{-1} \dot{U}_{L}^{\prime}(x) P_{L}(\cos \theta), \\
& F(\underbrace{}_{i})=\sum_{L=0}^{\infty} x^{-1} g_{L}(x) P_{L}(\cos \theta),
\end{aligned}
$$

one can write the partial wave equivalent of Eq. (8): 
- $39-$

$$
\begin{gathered}
U_{L}^{\prime \prime}+\left[k^{2}-L(L+1) x^{-2}-V_{1}\right] L_{L}^{\prime}=V_{2} g_{L}, \\
g_{L}^{\prime \prime}+\left[R^{22}-L(L+1) x^{-2}+N^{-1}\left(V_{2}-V_{3}-W+V_{N} x^{-1}\right)\right] g_{L} \\
-N^{-1} V_{N} g_{L}^{\prime}=N^{-1} V_{2} U_{L} .
\end{gathered}
$$

(Bia)

From Eq. (10) we obtain the dominant terms of the potentials near $x=0$. Representing each linearly independent solution as a vector $\psi=(U, g)$, we find two linearly independent regular solutions near zero:

$$
\begin{aligned}
& \psi_{L}^{(a)}(x)=\left(t_{L}, 0\right), \\
& \psi_{L}^{(b)}(x)=\left(0, t_{L}\right),
\end{aligned}
$$

where $t_{L} \approx x^{L}+1[I+x /(L+I)]$, pIus higher powers of $x$. Eq. (B2) is numerically integrated, using these starting forms, up to a point $\mathrm{x}=\overline{\mathrm{x}}$, and the general solution there is

$$
\psi_{L}(\bar{x})_{L}=A \psi_{L}^{(a)}(\bar{x})+B \psi_{L}^{(b)}(\bar{x})
$$


- $40-$

where $\mathrm{A}, \mathrm{B}$, are constants to be determined. For large distance we can rewrite Eq. (B2) using the asymptotic expression in Eq. (II). Retaining terms which fall off less rapidly than $x^{-3}$ we find

$$
U_{L}^{\prime \prime}+\left[k^{2}-L(L+1) x^{-2}\right] U_{L}^{\prime}=0
$$

(BSa)

$$
\begin{gathered}
g_{L}^{\prime \prime}-\frac{4}{x} g_{L}^{\prime}+\left[k^{2}-\frac{36}{43}+(4-L[L+1]) x^{-2}\right] g_{L} \\
=-\frac{36}{43} U_{L}^{\prime} .
\end{gathered}
$$

(Bub)

[We will discuss the $L=0, k=0$ case below.] Taking $x \geqq 20$ as a typical "asymptotic" distance, we see that the $\mathrm{x}^{-2}$ term is not neglibible for small $k(\approx 0.1)$ in $\mathrm{Eq} .(B 5 \mathrm{a})$, but is negligible in $\mathrm{Eq}$. (B5b). Of the four solutions to Eq. (B5), three are weil-behaved for $x \rightarrow \infty$. If we set $U_{I}=0$ in $\mathrm{Eq} .(\mathrm{B} 5 \mathrm{~b})$ and neglect $\mathrm{x}^{-2}$ terms, we find that the closed-channel function $g_{L}$ has two solutions,

$$
\begin{array}{r}
S_{I} \equiv e^{ \pm \gamma x}\left[1 \mp(\gamma x)+\frac{i}{3}(\gamma x)^{2}\right], \\
\left(\gamma=\left[\frac{36}{43}-k^{2}\right]^{1 / 2}\right) .
\end{array}
$$


$-41-$

The asymptotic regularity condition, Eq. ( $2 \mathrm{~b})$, requires

$$
\int_{0}^{\infty} d x g_{2}^{2}(x) N(x)<\infty,
$$

and since $N \sim x^{-4}, g_{L}$ must increase slower than $x^{3 / 2}$ for large $x$. Thus we are forced to drop the rising exponential form $\mathrm{S}+$ of Eq. (B6). The non-zero linearly independent solutions of $\mathrm{Eq}$. (BSa) are composed of the spherical Bessel functions:

$$
\begin{aligned}
U_{L} & =x j_{L}(k x), \quad \text { or } \\
& =x m_{L}(k x) .
\end{aligned}
$$

Corresponding to these open-channel solutions one finds a slowly converging 28 series of inverse powers for $g_{L}$. The three solutions for large $x$ are thus:

$$
\begin{aligned}
& \psi_{i}^{(c)}(x)=\left(0, s_{-}\right), \\
& \psi_{L}^{(d)}(x)=\left(x j_{L}, p_{L j}\right), \\
& \psi_{L}^{(e)}(x)=\left(x n_{L}, p_{L n}\right),
\end{aligned}
$$

(Ba)

where 
$-42-$

$$
\begin{aligned}
& p_{i 1}=x j_{L}\left[1+\frac{a}{x^{2}}+\frac{b}{x^{4}}+\right]+x n_{L}\left[\frac{c}{x}+\frac{d}{x^{3}} \cdots\right], \\
& p_{L n}=x m_{L}\left[1+\frac{\bar{a}}{x^{2}}+\frac{\bar{b}}{x^{4}}+\cdots\right]+x j_{L}\left[\frac{\bar{c}}{x}+\frac{\bar{d}}{x^{3}}\right]_{(B 10)}
\end{aligned}
$$

The coefficients in Eq. (BIO) can be obtained directly by use of Eq. (BF) and equating like powers of $x$. The result for $I=0$ is, for example,

$$
p_{o j}=\sin k x\left[1-\frac{3}{2}\left(\frac{43 k}{9 x}\right)^{2}+\cdots\right]-\cos k x\left[\frac{43 k}{9 x}+\cdots\right] \text {, }
$$

(BI)

$$
p_{0 n}=\cos k x\left[1-\frac{3}{2}\left(\frac{43 k}{9 x}\right)^{2}+\cdots\right]+\operatorname{sen} k x\left[\frac{43 k}{9 x}+\cdots\right] \text {. }
$$

Eq. (B2) is numerically integrated inward from some "asymptotic" point $\mathrm{x}=\mathrm{x}_{0}$, using these starting forms, down to the matching point $\mathrm{x}=\overline{\mathrm{x}}$, where the general solution is 


$$
\psi_{L}(\bar{x})>C \psi_{L}^{(c)}(\bar{x})+\psi_{L}^{(d)}(\bar{x})-\tan \delta_{i} \psi_{L}^{(e)} \psi_{L}(\bar{x})
$$

Setting $\psi_{L}(\bar{x})_{L}=\psi_{L}(\bar{x})_{\rangle}$and $\frac{d}{d x} \psi_{L}(\bar{x})_{L}=\frac{d}{d x} \psi_{L}(\bar{x})_{\rangle}$ gives four linear equations in the unknowns $A, B, C$ and $\tan \delta_{\mathrm{L}}$. We have tested the stability and consistency of the numerical procedure by comparing the results obtained for $x_{0}=20,25,30$ and $\bar{x}=2,3,4$, and find essentially no variation in the phase shifts for $\mathrm{L}=0$ (see Table IV). (An empirical correction for $\mathrm{L}=1$, obtained from our previous non-variational work was applied to account for the increase produced by the long-range $x^{-4}$ potential beyond $x=20$. For very low energies exact analytic expressions exist ${ }^{29}$ and are superior to our numerical results,

For $L=0$ and $k=0$ the long-range potential $V_{2}$ is not negligible in Eq. (B2a), even for $x \geq 20$, so it is not correct to use the asymptotic form Eq. (B5a). Instead, one has

$$
u_{0}^{\prime \prime}=-\frac{9}{2 x^{4}} g_{0}
$$




$$
g_{0}^{i i}-\frac{4}{x} g_{0}^{\prime}-\frac{36}{43}
$$

Solution $\psi_{0}(\mathrm{c})$ is unchanged from the form given in Eqs. (B9) and (B6), but the other two asymptotic solutions are now

$$
\begin{aligned}
& \psi_{0}^{(d)}=\left(x-\frac{9}{4 x}, x-\frac{253}{36 x}\right), \\
& \psi_{c}^{(e)}=\left(1-\frac{3}{4 x^{2}}, 1-\frac{3}{4 x^{2}}\right),
\end{aligned}
$$

and the linear combination shown in Eq. (B12) is unchanged except that $\tan \delta_{0}$ is replaced by the scattering length a. 
Footnotes and References

I. R. J. Drachman, Phys. Rev. I38, A1582 (1965)

2. R. J. Drachman, Phys. Rev. I44, 25 (I966)

3. H. Feshbach, Ann. Phys. (N. Y.) 5, 357 (1958)

4. A. Temkin and J. C. Lamkin, Phys. Rev. I2l, 788 (1961)

5. A. Dalgarno and N. Lynn, Proc. Phys. Soc. (London) A70, 223 (1957)

6. C. Schwartz, Phys. Rev. 124, 1468 (1961)

7. C. J. Kleinman, Y. Hahn, and I. Spruch, Phys. Rev. 140, A413 (1965)

8. A similar calculation [N. R. Kestner, J. Jortner, M. H. Cohen, and S. A. Rice, Phys. Rev. 140, A56 (1965)] with a less complete form of the potential gives qualitatively similar results

9. S. Marder, V. W. Hughes, C. S. Wu, and W. Bennett, Phys. Rev. 103,

1258 (1956); W. B. Teutsch and V. W. Hughes, Phys. Rev. 103, 1266 (1956)

10. Preliminary versions of this work have been reported previously:

a.) Bull. Am. Phys. Soc. 12, 70 (1967) and b.) Abstract Book, Fifth International Conference on the Physics of Electronic and Atomic Collisions. (Nauka, Leningrad, 1967) pp. 106-I08.

11. M. Gaylitis, Zh. Eksperim. i Teor. Fiz. 47, 160 (1964) [English transl: Soviet Phys. JETP 20, 107 (1965)]

12. P. G. Burke and A. J. Taylor [Proc. Phys. Soc. (London) 88, 549 (1966)] have shown that the orthogonality requirement requirement [Eq. (2a)] can be weakened.

13. T. Y. Wu and T. Ohmura, in Quantum Theory of Scattering, (PrenticeHaII, Inc., I962) pp. 57-68

14. The potentials $V_{I}$ and $V_{2}$ are expressible in closed form (Ref. 5). Their numerical evaluation thus served as a check on the accuracy of our computation. 
15. R. J. Drachman, Phys. Rev. 150, 10 (1966)

16. W. R. Falk, dissertation (University of British Columbia, 1965, unpublished), [See also W. R. Falk, P.H.R. Orth and G. Jones, Phys. Rev. Letters 14, 447 (1965)]. Other experimental values of $\mathrm{Z}_{\mathrm{k}}$ are: T. B. Daniel and R. Stump, Phys. Rev. 115, 1599 (1959) $\left[z_{k}=3.2\right]$; B. G. Duff and F. F. Heymann, Proc. Roy. Soc. (London) A270, 517 (1962) $\left[z_{k}=3.25 \pm 0.22\right]$; P. E. Osmon, Phys. Rev. I38, B216 (1965) $\left[\mathrm{z}_{\mathrm{k}}=2.46\right] ; \mathrm{T} . \mathrm{M} \cdot \mathrm{Kelly}$ and L. O. Roellig (Private Communication to P. Fraser) $\left[z_{k}=3.96 \pm\right.$ $0.04]$

17. Y. Hahn and L. Spruch, Phys. Rev. I40, AI8 (1965)

18. A. A. Frost, M. Inokuti and J. P. Lowe, J. Chem. Phys. 4I, $482(1964)$

19. B. H. Bransden, Proc. Roy. Soc. (London) A79, 190 (1962)

20. B. H. Bransden and Z. Jundi, Proc. Phys. Soc. (London) 22, 880 (1967); M. F. Fels and M. H. Mittleman, Phys. Rev. 163, $129(1967)$

21. C. J. Kleinman, Y. Hahn, and I. Spruch, Phys. Rev. 140, A413 (1965)

22. J. Callaway, R. W. LaBahn, R. T. Pu, and W. M. Duxler, Phys. Rev. (to be published).

23. A simple form which has proven to give fairly good results is $T(x)=1-B \exp \left(-m^{2}\right)$, with $B \approx 0.8$ and $m \approx 0.15$. This function does not, however, reproduce the maximum at $x=6$ shown in Fig. I. 24. R. Damburg and E. Karule, Proc. Phys. Soc. (Iondon) 90, 637 (1967) 25. J. F. Perkins, preprint (1967) 
26. L. I. Ponomarev, Zh. Eksperim. i Teor. Fiz. 22, 1549 (I967) [English transl: Soviet Physics-JETP 25, 1031 (1967)]

27. P. G. Burke and K. Smith, Rev. Mod. Phys. 34, 458 (1962) [See especially pp. 477-479]

28. Experience shows that problems of this type are not very sensitive to the detailed form of the closed-channel function, provided that the regularity condition (Eq. B7) is satisfied. In fact, if all the coefficients $(a, b, \ldots, \bar{a}, \bar{b}, \ldots)$ in Eq. (BIO) are omitted the phase shifts are unaffected, although the asymptotic oscillations of $\mathrm{g}_{\mathrm{L}}$ are not correctly represented.

29. T. F. O'Malley, L. Rosenberg and L. Spruch, Phys. Rev. 125, 1300 (I962) 


\begin{tabular}{llllllll}
$\mathrm{x}$ & \multicolumn{1}{c}{$\mathrm{V}_{1}$} & \multicolumn{1}{c}{$-\mathrm{V}_{2}$} & \multicolumn{1}{c}{$\mathrm{V}_{3}$} & \multicolumn{1}{c}{$\mathrm{N}$} & \multicolumn{1}{c}{$\mathrm{V}_{\mathrm{N}}$} & \multicolumn{1}{l}{${ }^{2}$} \\
0 & $2\left(\frac{1}{\mathrm{x}}-1\right)$ & 1 & $\frac{3}{2}\left(\frac{1}{\mathrm{x}}-1\right)$ & $3 / 4$ & $\frac{10}{3} \mathrm{x}$ & $6(1-2 \mathrm{x})$ \\
0.5 & 2.207 & $6.96(-1)$ & 1.229 & $5.38(-1)$ & $5.54(-1)$ & 1.913 \\
1.0 & $5.41(-1)$ & 3.99 & $1.57(-1)$ & 3.13 & 3.34 & $6.42(-1)$ \\
1.5 & 1.66 & 2.34 & $-1.40(-2)$ & 1.89 & 1.79 & 2.62 \\
2.0 & $5.49(-2)$ & 1.41 & -3.99 & 1.20 & 1.07 & 1.17 \\
2.5 & 1.89 & $8.48(-2)$ & -3.32 & $7.73(-2)$ & $6.84(-2)$ & $5.25(-2)$ \\
3.0 & $6.61(-3)$ & 5.14 & -2.18 & 4.96 & 4.41 & 2.28 \\
3.5 & 2.34 & 3.14 & -1.29 & 3.19 & 2.80 & $9.61(-3)$ \\
4.0 & $8.39(-4)$ & 1.95 & $-7.20(-3)$ & 2.06 & 1.77 & 3.96 \\
4.5 & 3.02 & 1.25 & -3.91 & 1.36 & 1.11 & 1.61 \\
5.0 & 1.09 & $8.19(-3)$ & -2.11 & $9.12(-3)$ & $7.04(-3)$ & $6.43(-4)$ \\
6.0 & $1.43(-5)$ & 3.86 & $-6.23(-4)$ & 4.43 & 2.98 & 1.00 \\
7.0 & $1.90(-6)$ & 2.03 & -2.00 & 2.36 & 1.38 & $1.53(-5)$ \\
8.0 & $2.53(-7)$ & 1.17 & $-7.18(-5)$ & 1.37 & $7.00(-4)$ & $2.31(-6)$ \\
9.0 & $3.38(-8)$ & $7.18(-4)$ & -2.91 & $8.47(-4)$ & 3.83 & $3.43(-7)$ \\
10.0 & $4.54(-9)$ & 4.67 & -1.31 & 5.52 & 2.24 & $5.06(-8)$
\end{tabular}

TABLE I. Numerical values for the potentials defined in Eq. (9), evaluated using the complete form of $G$ in elliptical coordinates. (Negative exponents appear in parenthesis in the usual way). 


\begin{tabular}{|c|c|c|}
\hline$E(e v)$ & A & $B$ \\
\hline 0 & 1.83 & 3.16 \\
\hline 0.54 & 1.65 & 2.79 \\
\hline 1.22 & 1.57 & 2.58 \\
\hline 2.17 & 1.51 & 2.43 \\
\hline 3.40 & 1.49 & 2.35 \\
\hline $4 \cdot 90$ & 1.49 & 2.31 \\
\hline 6.66 & 1.52 & 2.30 \\
\hline 8.70 & 1.57 & 2.33 \\
\hline 11.0 & 1.62 & 2.38 \\
\hline 13.6 & 1.68 & 2.44 \\
\hline 17.8 & 1.78 & 2.54 \\
\hline
\end{tabular}

TABLE II. Positron-Helium Annihilation Rate $\frac{1}{2} \mathrm{Z}_{\mathrm{k}}$. Column A gives the present results, and Column $B$ is the non-variational result of Ref. 15. The Dirac rate is $\frac{I}{2} Z_{k}=1$. 


$\begin{array}{lll}\mathrm{v}=\beta \mathrm{x} & \mathrm{M} & \mathrm{Y} \\ 0 & 1.67 & 1 \\ 0.5 & 1.68 & 1.91 \\ 1.0 & 1.83 & 2.78 \\ 1.5 & 2.13 & 3.50 \\ 2.0 & 2.55 & 4.07 \\ 2.5 & 3.07 & 4.53 \\ 3.0 & 3.66 & 4.92 \\ 3.5 & 4.29 & 5.25 \\ 4.0 & 4.90 & 5.55 \\ 4.5 & 5.47 & 5.82 \\ 5.0 & 5.97 & 6.06\end{array}$

TABLE III. Correlation functions for $\mathrm{e}^{+}-$Helium annihilation. $M$ is used in the present work and is defined in Eq. (28). $Y \equiv[I+G(x, x)]^{2}+\left\langle G^{2}\right\rangle$ is used analogously in the non-variational work (Ref. 15), and employs full monopole suppression in G. 
$\tan \delta(k=0.1)$

$\overline{\mathbf{x}}$

$x_{0}=20$

$\mathbf{x}_{0}=25$

$x_{0}=30$

2

0.12753

0.12804

0.12808

3

0.12747

0.12798

0.12803

4

0.12675

0.12726

0.12731

$\tan \delta(k=0.7)$

$\bar{x}$

$x_{0}=20$

$x_{0}=25$

$x_{0}=30$

2

$-0.07393$

$-0.07386$

$-0.07383$

3

$-0.07396$

$-0.07389$

$-0.07386$

4

$-0.07440$

$-0.07433$

$-0.07430$

TABLE IV. S-wave $e^{+}-H$ results. The sensitivity of $\tan \delta$ to matching radius $(\bar{x})$ and asymptotic radius $\left(x_{0}\right)$ is shown, for two values of positron momentum $k$. 


$\begin{array}{cccccc}\mathrm{k} & \tan \delta & \tan \delta_{\mathrm{S}} & \tan \delta_{\mathrm{H}} & \mathrm{Q} & \mathrm{Q}_{\mathrm{Hs}} \\ 0 & -1.85 & -2.10 & 0.582 & 0.91 & \\ 0.1 & 0.128 & 0.152 & -0.058 & 0.89 & \\ 0.2 & 0.158 & 0.190 & -0.116 & 0.90 & 0.87 \\ 0.3 & 0.135 & 0.170 & -0.170 & 0.90 & \\ 0.4 & 0.089 & 0.121 & -0.222 & 0.91 & 0.89 \\ 0.5 & 0.034 & 0.062 & -0.270 & 0.92 & \\ 0.6 & -0.022 & 0.007 & -0.314 & 0.91 & 0.89 \\ 0.7 & -0.074 & -0.054 & -0.354 & 0.93 & \end{array}$

TABLE V. S-wave $e^{+}-H$ scattering results. The entries without subscripts refer to the present work, and are averages over $\bar{x}$. The other phase shifts are: $\delta_{\mathrm{S}}$-Schwartz (Ref. 6); $\delta_{\mathrm{H}}$-Hartree; and $\delta_{\mathrm{HS}}-\mathrm{Hahn}$ and Spruch (Ref. 16). The figure of merit $\mathrm{Q}$ is defined in the text. For $\mathrm{k}=0$, scattering lengths are given. 


$\begin{array}{lcccc}\mathrm{k} & \tan \delta & \tan \delta_{\mathrm{H}} & \tan \delta_{\mathrm{NV}} & \mathrm{Q} \\ 0 & -0.511 & 0.420 & -0.659 & 0.86 \\ 0.1 & 0.036 & -0.042 & 0.050 & 0.85 \\ 0.2 & 0.047 & -0.083 & 0.072 & 0.84 \\ 0.3 & 0.039 & -0.124 & 0.071 & 0.84 \\ 0.4 & 0.020 & -0.163 & 0.056 & 0.84 \\ 0.5 & -0.007 & -0.203 & 0.032 & 0.83 \\ 0.6 & -0.039 & -0.240 & 0.002 & 0.83 \\ 0.7 & -0.073 & -0.278 & -0.031 & 0.83 \\ 0.8 & -0.107 & -0.313 & -0.066 & 0.83 \\ 0.9 & -0.142 & -0.346 & -0.100 & 0.83 \\ 1.0 & -0.176 & -0.379 & -0.134 & 0.83 \\ 1.1 & -0.208 & -0.409 & -0.168 & 0.83 \\ 1.145 & -0.223 & -0.423 & -0.182 & 0.83\end{array}$

TABLE VI. S-wave $e^{+}-$He scattering results. The entries without subscripts refer to the present work and are averages over $\bar{x}$. The other phase shifts are: $\delta_{\mathrm{H}}$ - Hartree; $\delta_{\mathrm{NV}}$ - nonvariational (Ref. 2). For $k=0$, scattering lengths are given. $Q$ is computed assuming $\delta_{\mathbb{N V}}$ to be exact. 


$\begin{array}{llll}\mathrm{k} & \tan \delta_{1} & \tan \delta_{1}(\mathrm{LB}) & \tan \delta_{1} \text { (ext) } \\ 0.1 & 0.0073(1) & 0.0082 & 0.0086(1) \\ 0.2 & 0.0263(2) & 0.0289 & 0.032(1) \\ 0.3 & 0.0518(5) & 0.0548 & 0.066(4) \\ 0.4 & 0.0764(9) & 0.0801 & 0.11(1) \\ 0.5 & 0.0952(14) & 0.0994 & 0.14(1) \\ 0.6 & 0.106(2) & 0.112 & 0.17(2) \\ 0.7 & 0.109(2) & 0.119 & 0.19(2)\end{array}$

TABLE VII. P-wave $e^{+}-H$ scattering results. The present results (corrected for long-range effects as described in Appendix B) are compared with the lower bounds (LB) and extrapolated values (ext), obtained in Ref. 21. The estimatederror in the last digit is shown in parenthesis. 


\section{Figure Caption}

Figure 1. Zero-energy scattering solutions for $e^{+}-H$. The functions $X$ and $F$ are open-channel and closed-channel functions, and $T=F / X$ is the suppression factor. The asymptotic normalization is $X=1-a / x, F=X$ and $T=1$ 
PRECEDNG PAGE BLANK NOT FILMED.

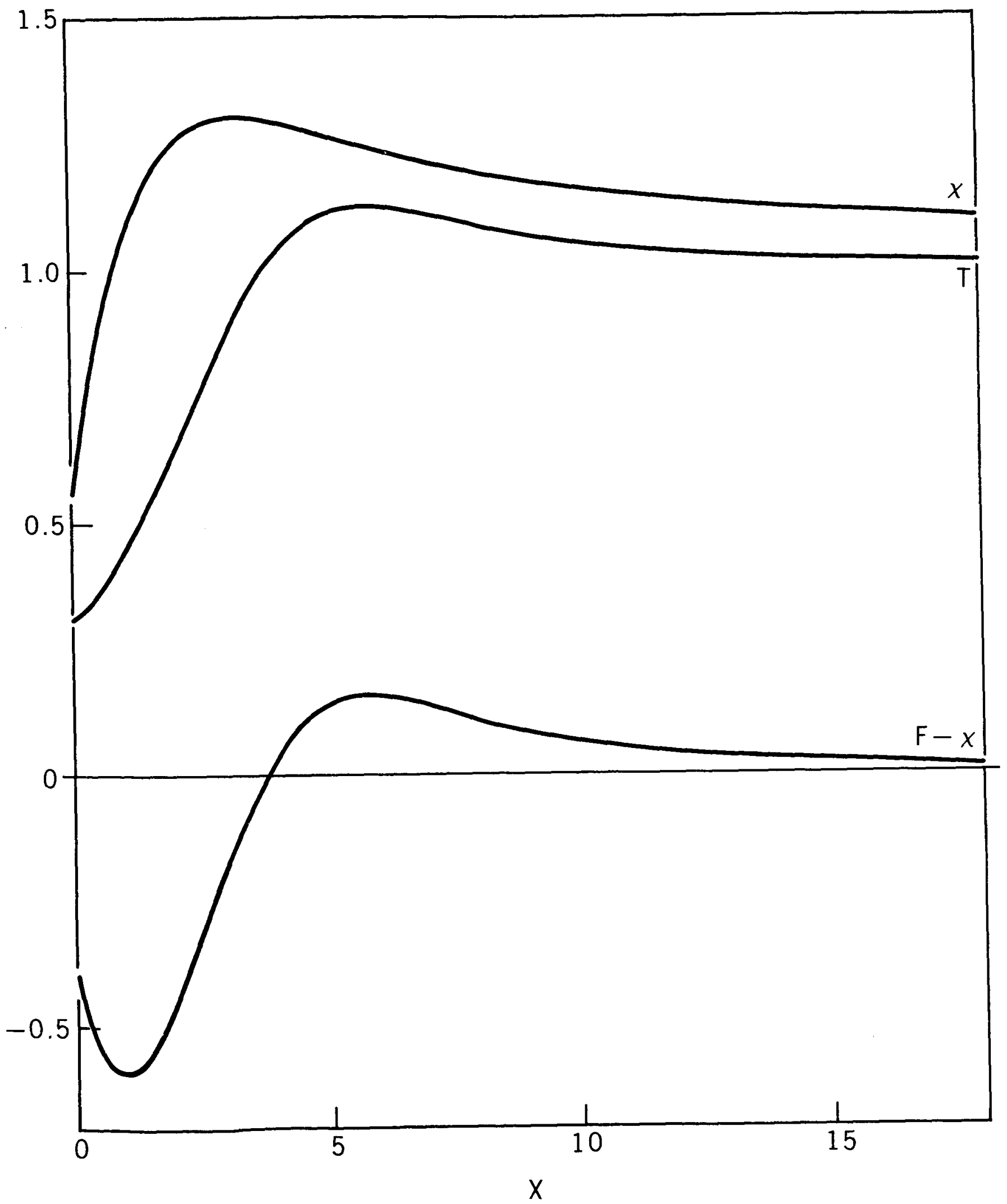

